Proceedings of the International Symposium on Physics of Materials (ISPMA 14), September 10-15, 2017, Prague

\title{
Experimental Observations and Modeling of Localization in Superelastic NiTi Polycrystalline Alloys: State of the Art
}

\author{
M. Frost ${ }^{a, b, *}, \mathrm{P} . \mathrm{SEDLÁK}^{a, b}$ AND T. Ben ZineB ${ }^{d, e}$ \\ ${ }^{a}$ Nuclear Physics Institute of the CAS, Rež 130, Rež, CZ-25068, Czechia \\ ${ }^{b}$ Institute of Thermomechanics of the CAS, Dolejškova 5, Praha, CZ-18200, Czechia \\ ${ }^{c}$ Université de Lorraine, CNRS, Arts et Métiers ParisTech, LEM3, F-54000 Nancy, France
}

\begin{abstract}
We briefly review the recent experimental and theoretical research on the modeling of localized deformation in NiTi shape memory alloys. These alloys exhibit a remarkable response to mechanical loading with a relatively large reversible strains called superelasticity. We point up factors affecting formation, topology and propagation of localization patterns. These factors bring considerable difficulties to the development of constitutive models and superimpose with theoretical and practical issues related to the modeling of material instability. Some of the models are briefly introduced with a particular attention paid to characteristics of underlying theoretical frameworks.
\end{abstract}

DOI: 10.12693 /APhysPolA.134.847

PACS/topics: $62.20 . \mathrm{fg}, 46.32 .+\mathrm{x}$

\section{Introduction}

The effect when in spite of monotonic loading deformation concentrates into a relatively narrow region of the structure is usually termed localization of deformation (or strain localization). The deformation response of the rest of the structure then evolves much less (often remains rigid or even unloads), hence heterogeneity of mechanical state of the material increases. The effect manifests itself as shear bands (e.g. metals, concrete, sands), kink bands (fiber-reinforced ceramics), crack bands, necking, etc. With increasing loading, localization often leads to damage and, ultimately, to failure of the structure.

Because of apparent practical importance, first serious attempts to model localization can be traced back to the beginning of the twentieth century [1]; however, significant progress is related to advancement of technical computing in the last quarter of the century, when a wide class of so-called nonlocal continuum theories appeared [2-4]. In these theories, mechanical (and thermodynamic) response of a material point is not uniquely determined by values of state and internal variables (fields) in that point only, but state of material points in a vicinity is also taken into account. The initial aim (common also to some other generalized continuum theories) was to enrich the "classical" continuum by some information below its resolution level. Particular formulations usually stem from considerations on characteristic material lengths with respect to "wave-lengths" of expected deformation fields and relations between internal scales of material and computational domain scaling. In the context of the modeling of localization, nonlocal enrichments regularize strain-softening models and prevent spurious concentration of strain to infinitely small regions $[5,6]$.

\footnotetext{
*corresponding author; e-mail: mfrost@email.cz
}

Shape memory alloys (SMAs) are intermetallic materials exhibiting a reversible solid-to-solid phase transformation. The phase change between austenite and martensite may be induced by temperature changes and/or deformation. If a piece of the alloy is deformed at a high enough temperature, it recovers its original shape after unloading even if deformation reaches several percent; this effect is called superelasticity (also pseudoelasticity) and the related (recoverable inelastic) strain linked with the phase transformation is termed transformation strain. In fact, martensitic phase features several (non-elastic) structural mechanisms which allow for recoverable accommodation of (relatively large) imposed strains; they are collectively termed reorientation (processes) hereinafter. ${ }^{\dagger}$

Having found many applications in medicine, civil engineering or aerospace industry [7], NiTi-based alloys are a prominent class within SMAs usually utilized in the form of thin structures, e.g. wires, strips or plates. In such structures, the martensitic transformation often does not occur in a spatially homogeneous manner; instead, localized "martensitic bands" form within the austenitic sample and their fronts propagate leaving a material with modified dimensions and microstructure (phase) behind. The macroscopic picture is very similar to the localization of plastic deformation in certain steels and alloys well-known as Lüders bands (also slip bands): the onset is usually accompanied by a stress overpeak and followed by a stress plateau, material rehardens after exhausting the available portion of inelastic strain and the formation of bands depends on microstructural properties of the material. There are also some specific features of NiTi alloys: (a) the attainable inelastic (transformation) strain is reversible and

\footnotetext{
${ }^{\dagger}$ As common in metals, however, if the mechanical loading is too severe, processes linked with plasticity are initiated and the original shape is not fully recovered.
} 
strongly loading mode-dependent [8], (b) physical properties of the phases differ pronouncedly and transformation precursor effects are well-documented [9], (c) microstructural aspects of martensitic transformation in polycrystalline SMA (e.g. compatibility and interactions of phases and/or martensitic variants in severely constrained polycrystalline environment) are rather complex and not fully understood yet.

This short contribution aims to summarize recent progress in both experimental investigation and computational modeling of such localization in NiTi SMA and point out some related peculiarities.

\section{Experimental observations}

Vast majority of experiments were performed with superelastic NiTi alloy at constant ambient temperature so that mechanical loading induces distinct phase transformation(s) between phases. In their pioneering works, Shaw and Kyriakides [10, 11] observed localized tensile deformation on NiTi thin strips covered with a brittle oxide coating. When subjected to straining, coating shattered allowing observation of localized deformation by the naked eye. Substantial changes of temperature documented by complementary thermal camera images confirmed a tight relation of this localization to martensitic transformation, which is accompanied by release/absorption of a latent heat. Because of a strong coupling between stress and temperature in the thermodynamic phase stability criterion (Clausius-Clapeyron type), the work also showed strong dependence of localization pattern (number of bands) on deformation rate. The dynamics of formation, propagation and coalescence of transformation bands and the relation to the strainrate was further studied by an in-situ infrared camera in $[12,13]$ or by digital image correlation (DIC) method in [14]. Next, we restrict the review to quasistatic experiments (low loading rates, close to isothermal conditions) in which the influence of the transformation heat to the localization process is minimized.

Localization during superelastic loading has also been extensively documented in thin-walled NiTi tubes. Sun and $\mathrm{Li}$ [15] were first to show that spiral bands may form in tension of the tubes. Further studies [16, 17] showed that different topologies of localization patterns can emerge depending e.g. on the testing temperature. The development of propagating localized deformation in tension and homogeneity of deformation in compression were later documented with DIC in [18-20]. Consequently, bent tubes exhibit wedge-like localization structures on the outer surface (deformed in tension) whereas homogenous deformation on the inner one (deformed in compression) [19, 20]. Interesting findings have been brought by the very recent experiments of combined loading of tubes: Reedlunn and coworkers [21] performed a set of axial-torsion experiments following both proportional and non-proportional strain paths. They confirmed presence of propagating transformation fronts in pure tension, their absence in pure torsion, and a progression of behaviors in between as reported in [15]. Moreover, they first reported vertical columns of shear strain to gradually appear and disappear during torsioninduced transformation, suggesting a kind of nonpropagating localization develops. Proportional loading of tubes in combined axial and radial stress (realized by internal pressure) has been examined by [22] also with DIC. Localized helical bands with inclinations dependent on the stress ratio formed except for loading modes close to equibiaxial tension; in that case the material exhibited hardening and homogeneous deformation.

Pure tension tests on tubes were repeated by [23] using a sophisticated experimental design (minimizing bending and torsion moments that might be induced in the tube during its tension). They made assertions that can be generalized as follows (see also [24, 25]): (i) stress peaks on the stress-strain curves are often not indicating nucleation of phases; they are rather connected with formation of bands, which, however, separate regions with distinct dominant phases, (ii) the stress plateau on the stressstrain curve is only a manifestation of the localization of deformation; transformation processes can occur before and after this loading stage as well, (iii) localization can occur even if the macroscopic tensile stress-strain curve does not exhibit a clear stress peak and plateau. The authors also suggested that the "structure-material coupling" can strongly influence evolution of domain morphologies; e.g. compare helical bands in $[18,23]$ with branched "finger-structured" bands observed in [16, 19] or with cylindrical (ring) domains in $[16,17]$ in case of NiTi tubes or planar fronts $[13,26]$ with "criss-cross" patterns $[11,27]$ in NiTi strips.

In addition to wires, thin strips (ribbons) and thinwalled tubes (where at least one dimension is substantially lower than the largest one), localization has also been documented on specimens with all dimensions comparable. For instance, Elibol et al. [28] confirmed homogeneity of compressive deformation on a cylindrical specimen (with the diameter equal to the height) and localization in a rod-like specimen subjected to tension. Let us note that some authors $[18,29]$ suggested determining influence of (manufacturing) texture (usually present in wires, rolled sheets, etc.) on this asymmetric behavior due to the favorable partitioning of strains, stresses and phases among grains and the cooperative and catalytic manner of propagation of transformation across neighboring grains.

Localization is very common in superelastic NiTi wires loaded in tension, see e.g. the investigation of influence of two-stage transformation via R-phase in [30]. Propagation of deformation bands is optically observable as a change of the wire diameter behind band fronts. The front itself has been investigated by Young and coworkers [31] with in-situ synchrotron X-ray diffraction methods during loading of an ultrafine-grained NiTi (about $50 \mathrm{~nm}$ grain size). It was found that within the transformation zone of finite depth, both phases are highly 
strained and only a small amount of residual austenite remains within the "martensitic" band. This contrasts with works $[32,33]$ which reported significant remnants of austenite within the band in coarse-grained $(50-200 \mu \mathrm{m})$ strip specimens. The different grain size suggests a strong influence of this parameter to localization characteristics; indeed, determining influence of grain size to the superelastic response was shown in [34, 35]. Even more detailed picture of the band front was brought by $3 \mathrm{D}$ X-ray synchrotron diffraction measurements by Sedmák et al. [36]. Advanced tomographic techniques allowed to resolve elastic strains and stresses in approx. 15,000 austenitic grains $(6 \mu \mathrm{m})$ at and close to localized transformation front in a superelastic NiTi wire under tension. They were able to partially reconstruct its topology ("a nose-cone shape") and found that the internal stress states in grains massively change and redistribute within the propagating front so that, at the onset of transformation, austenitic grains were (in average) exposed to equivalent stresses more than $200 \mathrm{MPa}$ higher then externally measured plateau stress. The results suggest that the severe changes of stress states ahead the (localized) phase interface due to the macroscopic internal stress prevail over the local effects of grain orientation, size and neighborhood during formation and propagation of the transformation front in this case.

In spite of substantial progress brought by the above-mentioned and other microstructural observations (e.g. [18] using electron backscatter diffraction (EBSD) technique, [37] employing combination of DIC and scanning electron microscopy), the particular phase transformation-based microstructural phenomenon leading to Lüders-like behavior in polycrystalline NiTi (i.e. the counterpart to dynamic strain aging mechanism stimulating conventional Lüders bands in mild steels) has not been fully established yet. It is expected that the key is the understanding of interactions at the mesoscopic level, i.e. the level of transforming grains and their aggregates. Fortunately, the subduing influence of the macroscopic internal stress reported by Sedmák [36] favors macroscopic perception of Lüders-like behavior in NiTi as a mechanical phenomenon (see also reasoning by Liu [24]) tractable (for a "fixed material") by means of modern tools of structural mechanics as outlined in the next section.

\section{Modeling}

Despite abundance of constitutive models of SMAs in the literature - originating at different scales of description and simplification, see recent reviews [38, 39] macroscopic (continuum) models covering localization in polycrystalline SMA have been attempted rarely.

Significant effort has been devoted to transferring the extensive knowledge gained from experimental and theoretical studies on single-crystals to the modeling of polycrystalline SMA. However, the academic search for an effective, plausible and physically realistic micro scale- macro scale transition model continues. A somehow simplistic combination of phase field approach with a strainsoftening response regularized by a rate-dependent transformation kinetics in [40] is one of few models of this kind incorporating also the localization phenomenon.

Macroscopic models stemming from continuum mechanics and thermodynamics are slightly more numerous, likely since complex interactions leading to localization can be merged and distilled to averaged contributions to the constitutive laws in a more phenomenological way. ${ }^{\ddagger}$ In the pioneering works $[11,41]$, Shaw et al. used a simple isotropic plasticity model with strain-softening to study localization in NiTi strips deformed in pure tension. They were able to capture some basic features of forming localization bands and to study influence of latent heat via incorporation of the thermal coupling. The concept was extended for unloading in [42]. These simple models adopted isotropic plasticity-based constitutive laws disregarding specifics of SMA material. A certain remedy came with one-dimensional phenomenological models for SMA wires [43, 44] with incorporated specific chemical free energy of transformation, refined transformation kinetics, and strain softening. Although internal variables related to the phase fraction of martensite are introduced, regularization of material instability is incorporated via adaptation of strain-gradient elasticity approach; consequently, the displacement field is interpolated with (cubic) Hermite polynomials in the finite element implementation of the models.

However, the motivation for including higher gradients of (total) strain can be questioned and this approach requires modification of global balance laws and utilization of interpolants with higher continuity, which is computationally more demanding, especially if the formulation of the model would expand to three-dimensional setting. A useful alternative may be to link localization to some internal variable(s) related to phase transformation. ${ }^{\S}$ Regularization of the model is achieved via incorporating information on values of a chosen internal variable in its close neighborhood. This may be performed in three steps: (i) introducing a "nonlocal counterpart" to some (physically motivated) internal variable, which encompass the information, (ii) relating these "twin" variables (nonlocal variable is computed from the local one), and (iii) modifying the constitutive laws. The first and the last steps provide enough space for development of new constitutive laws for a particular material. In the second step, two well-established approaches can be borrowed from structural mechanics: the implicit nonlocal gradient approach (iNGA) and the nonlocal integral ap-

\footnotetext{
$\ddagger$ Let us remind that this class of models does not aim to reconstruct particular martensitic microstructures.

$\S$ Internal variables are a powerful tool for capturing inelastic deformation in constitutive laws. They represent the microstructural state of the material and enter the formulae for stored and dissipated energy.
} 
proach (NIA). In the former case, the local and corresponding nonlocal variables are linked via an additional (elliptic) partial differential equation (PDE), in the latter case, these variables are linked via an integral relation - the nonlocal variable in a material point is defined as a weighted integral average of the local one (usually, the weight function is concentrated in a close neighborhood of the point). Corresponding general mathematical formulations are closely related; specifically, iNGA can be derived from NIA using particular weighting functions [45]. Moreover, an internal parameter related to some characteristic length scale is present in both approaches and determines the extent of the affecting neighborhood.

The model of Armattoe et al. [46] implemented in the two-dimensional setting is an example of the iNGA. It refines and extends the superelastic localization model of Duval et al. [47] for any (quasistatic) thermomechanical loading. Two local internal variables — a scalar representing volume fraction of martensite and a tensor of transformation strain of martensite - are introduced and the scalar one also has its nonlocal counterpart. The non-local variable modifies the critical driving force for transformation, i.e. the "shape of (transformation) yield surface", and allows for strain-softening material response. The nonlocal variable does not appear in the free energy, which is adopted from the (strictly local) SMA model [48]. The PDE relating local and nonlocal variables is accompanied by the homogeneous Neumanntype of boundary condition; it also assures that the total amount of the nonlocal volume fraction of martensite in the domain is at any time equal to the local one. Necessity of solving an additional PDE on the computational domain leads to an additional degree of freedom in the finite element implementation of the model.

A nonlocal modification of the local three-dimensional model by Sedlák et al. [49, 50] presented in [51] and utilized for the computational reconstruction of the martensite band front in [36] is an example of the NIA. The same two local and one (scalar) nonlocal internal variables as in [46] are introduced. However, the nonlocal variable appears only in the free energy (in the phase-interaction term) and not in the dissipation function, which directly determines the yield criteria in the model. $\mathbb{1}$ The normalized Gauss distribution function is employed as the kernel of the integral operator; its standard deviation serves as the internal length parameter. The retarded step technique (staggered computation of nonlocal variable) with restricted time stepping is used in the finite element implementation to reduce computational costs.

Two other conventional local constitutive SMA models have been also modified for localization via strainsoftening recently; both of them utilize nonlocal variable

ILet us note that a special, asymmetric coupled form of the dissipation function forms the core of the NiTi-tailored constitutive model of Sedlák et al. derived from volume fraction of martensite. Ahmadian et al. [52] modified the free energy of the model [53] and NIA is suggested for regularization, although the final form of constitutive equations is not explicitly stated in the paper. The original model of Brinson [54] was modified in [55] in the expression of the critical driving force and resulting strain-softening is regularized by iNGA. However, because of a simplistic formulation of the original models (surpassed by later works of the same and other authors), the ability of their nonlocal derivatives to plausibly predict SMA behaviors is rather limited.

\section{Discussion and concluding remarks}

As apparent from the previous subsection, the combination of dependency on geometry of the specimen, its microstructure, applied loading mode and boundary conditions hinders sound understanding of the physical roots of the phenomenon and makes any attempt for a (reasonably) general modeling description a real challenge.

Many rather fundamental questions have not been answered yet, e.g.: Which microstructural level and what type of mismatch is key for the material instability? Does localization occur during reorientation of martensite as indicated by experiments of Liu et al. $[56,57]$ or this is not the case as observed in [17]. Could some characteristics of the morphology of the localization band front revealed by Sedmák et al. [36] in wires subjected to tension be somehow generalized also for other geometries and loading modes? Is the strain-softening rooted in the stored or dissipated energy of the material?

From the modeling point of view, resolving the first two problems will help to set up effective internal variables; all reviewed models link the softening simply to the scalar variable representing volume fraction of martensite, which may be insufficient for capturing loadingmode dependent localization patterns observed in experiments (cf. bending of a SMA tube simulated in [55]). It can also hint at physical-based determination of the internal length parameter (which is a problem for some other nonlocal continuum models, too); this is also related to the third question. The last question is strongly related to the modeling of dynamics of the material in which thermal coupling plays an important role (e.g. strain dependence of localization).

Other difficulties are related to fitting of the strainsoftening response. Since softening is "invisible" in macroscopic stress-strain curves with a plateau, the phase transformation starts before onset of the stress overpeak and it is often incomplete at the end of the plateau [23], dedicated experiments revealing the course of the strain-softening are needed." Quantitative results

$\|$ Let us note that careful determination of elastic properties of martensite in [58] can help to clarify the further increase of recoverable strain during loading beyond macroscopic transformation plateau [25]. 
have been reached recently in [59] via a sophisticated measurement of a NiTi-stainless steel composite and by Alarcon et al. [60] via a special geometry of NiTi bulk specimen.

Finally, a weak point of most of the nonlocal continuum models is the justification of the treatment of the domain boundary. Neither modification of the weight function close to it in NIA, neither the requirements leading to Neumann boundary condition in iNGA are clearly motivated in most of them. This remains valid also for the above-mentioned SMA models.

\section{Acknowledgments}

This work has been supported by the OP RDE, Ministry of Education, Youth and Sports of the Czech Republic under the project ESS SCANDINAVIA CZ.02.1.01/0.0/0.0/16_013/0001794 and by the Czech Science Foundation under the project AdMat, No. 14$36566 \mathrm{G}$.

\section{References}

[1] E. Cosserat, F. Cosserat, Théorie des corps déformables, Herrman, Paris 1909.

[2] A.C. Eringen, Int. J. Eng. Science 19, 1461 (1981).

[3] Z.P. Bažant, F.B. Lin, Int. J. Num. Methods Eng. 26, 1805 (1988).

[4] P.A. Vermeer, R.B.J. Brinkgreve, in: Localisation and Bifurcation Theory for Soils and Rocks, Eds. R. Chambon, J.Desrues, I.Vardoulakis, Balkema, Rotterdam 1994, p. 89.

[5] Z.P. Bažant, M. Jirásek, J. Eng. Mech. 128, 1119 (2002).

[6] M. Jirásek, S. Rolshoven, Int. J. Eng. Sci. 41, 1553 (2003).

[7] J. Mohd Jani, M. Leary, A. Subic, M.A. Gibson, Mater. Des. 56, 10781113 (2014).

[8] K. Gall, H. Sehitoglu, Y.I. Chumlyakov, I.V. Kireeva, Acta Mater. 43, 1203 (1999).

[9] P. Šittner, M. Landa, P. Lukáš, V. Novák, Mech. Mater. 38, 475 (2006).

[10] J.A. Shaw, S. Kyriakides, Acta Matert. 45, 683 (1997).

[11] J.A. Shaw, S. Kyriakides, Int. J. Plast. 13, 837 (1997).

[12] E.A. Pieczyska, H. Tobushi, K. Kulasinski, Smart Mater. Struct. 22, 035007 (2013).

[13] X. Zhang, P. Feng, Y. He, T. Yu, Q. Sun, Int. J. Mech. Sci. 52, 1660 (2010).

[14] C. Elibol, M.F.X. Wagner, Mat. Sci. Eng. A 643, 194 (2015).

[15] Q.P. Sun, Z.Q. Li, Int. J. Solids Struct. 39, 3797 (2002).

[16] P. Feng, Q. Sun, J. Mech. Phys. Solids 54, 1568 (2006).

[17] K.L. Ng, Q. Sun, Mech. Mater. 38, 41 (2006).

[18] S.C. Mao, J.F. Luo, Z. Zhang, M.H. Wu, Y. Liu, X.D. Han, Acta Mater. 58, 3357 (2010).
[19] B.J. Reedlunn, C.B. Churchill, E.E. Nelson, J.A. Shaw, S. Daly, J. Mech. Phys. Solids 63, 509 (2014).

[20] N.J. Bechle, S. Kyriakides, Int. J. Solids Struct. 51 , 967 (2014).

[21] B. Reedlunn, J.A. Shaw, S. Daly, in: Conf. Proc. from the Int. Conf. on Shape Memory and Superelastic Technologies, San Diego, CA 2017, 43917.

[22] N.J. Bechle, S. Kyriakides, Int. J. Plast. 82, 1 (2016).

[23] D. Favier, H. Louche, P. Schlosser, L. Orgeas, P. Vacher, L. Debove, Acta Mater. 55, 5310 (2007).

[24] Y. Liu, Mat. Sci. Eng. A 271, 506 (1999).

[25] G. Tan, Y. Liu, P. Šittner, M. Saunders, Scr. Mater. 50, 193 (2004).

[26] D. Jiang, S. Kyriakides, C.M. Landis, K. Kazinakis, Eur. J. Mech A Solids 131, (2017).

[27] G. Murasawa, S. Yoneyama, T. Sakuma, Smart Mater. Struct. 16, 160 (2007).

[28] C. Elibol, M.F.X. Wagner, Mat. Sci. Eng. A 621, 76 (2015).

[29] P. Šittner, Y. Liu, V. Novák, J. Mech. Phys. Solids 53, 1719 (2005).

[30] Y. Xiao, P. Zeng, L. Lei, Mater. Res. Express 3, 105701 (2016).

[31] M.L. Young, M.F.X. Wagner, J. Frenzel, W.W. Schmahl, G. Eggeler, Acta Mater. 58, 2344 (2010).

[32] J.C. Brinson, I. Schmidt, R. Lammering, J. Mech. Phys. Solids 52, 1549 (2004).

[33] M. Kimiecik J.W. Jones, S. Daly, Materials Letters 95, 25 (2013).

[34] R. Delville, B. Malard, P. Šittner, D. Schryvers, Acta Mater. 58, 4503 (2010).

[35] A. Ahadi, Q. Sun, Acta Mater. 76, 186 (2014).

[36] P. Sedmák, J. Pilch, L. Heller, J. Kopeček, J. Wright, P. Sedlák, M. Frost, P. Šittner, Science 353, 559 (2016).

[37] M. Kimiecik, J.W. Jones, S. Daly, Acta Mater. 94 214 (2015).

[38] C Cisse, W Zaki, T. Ben Zineb, Int. J. Plast. 76, 244 (2016).

[39] C Cisse, W Zaki, T. Ben Zineb, Smart Mater. Struct. 25, 103001 (2016).

[40] A.V. Idesman, V.I. Levitas, D.L. Preston, J.-Y. Cho, J. Mech. Phys. Solids 53, 495 (2005).

[41] M.A. Iadicola, J.A. Shaw, Int. J. Plast. 20, 577 (2004).

[42] B. Azadi, R.D. Rajapakse, D.M. Maijer, Int. J. Solids Struct. 44, 6473 (2007).

[43] J.A. Shaw, Int. J. Plast. 16, 541 (2000).

[44] B.-C. Chang, J.A. Shaw, M.A. Iadacola, Continuum Mech. Thermodyn. 18, 83 (2006).

[45] R.H.J. Peerlings, M.G.D. Geers, R. de Borst, W.A.M. Brekelmans, Int. J. Solids Struct. 38, 7723 (2001).

[46] K.M. Armattoe, M. Haboussi, T. Ben Zineb, Int. J. Solids Struct. 51, 1208 (2014). 
[47] A. Duval, M. Haboussi, T. Ben Zineb, Int. J. Solids Struct. 48, 1879 (2011).

[48] Y. Chemisky, A. Duval, E. Patoor, T. Ben Zineb, Mech. Mater. 43, 361 (2011).

[49] P. Sedlák, M. Frost, B. Benešová, T. Ben Zineb, P. Šittner, Int. J. Plast. 39, 132 (2012).

[50] M. Frost, B. Benešová, P. Sedlák, Math. Mech. Solids 21, 358 (2016).

[51] M. Frost, P. Sedlák, P. Šittner, Solid State Phenom. 258, 141 (2016).

[52] H. Ahmadian, S.H. Ardakani, S. Mohammadi, Int. J. Solids Struct. 63, 167 (2015).

[53] J.G. Boyd, D.C. Lagoudas, Int. J. Plast. 12, 805 (1996).
[54] L.C. Brinson, R. Lammering, Int. J. Solids Struct. 30, 3261 (1993).

[55] H. Badnava, M. Kadkhodaei, M. Mashayekhi, J. Intell. Mater. Syst. Struct. 26, 2531 (2015).

[56] Y. Liu, Y. Liu, J. Van Humbeeck, Acta Mater. 47, 199 (1998).

[57] Y. Liu, Z. L. Xie, J. Van Humbeeck, L. Delaey, Acta Mater. 47, 645 (1999).

[58] P. Sedlák, M. Frost, Acta Phys. Pol. A 134, 842 (2018).

[59] J.F. Hallai, S. Kyriakides, Int. J. Plast. 47, 1 (2013).

[60] E. Alarcon, L. Heller, S.A. Chirani, P. Šittner, J. Kopeček, L. Saint-Sulpice, S. Calloch, Int. J. Fatigue 95, 76 (2017). 